\title{
Docking of Small Ligands to Low-Resolution and Theoretically Predicted Receptor Structures
}

\author{
MAREK WOJCIECHOWSKI, JEFFREY SKOLNICK \\ Laboratory of Computational Genomics, Donald Danforth Plant Science Center, \\ 893 North Warson Rd., St. Louis, Missouri 63141
}

Received 25 April 2001; Accepted 5 June 2001

\begin{abstract}
We have developed a simple docking procedure that is able to utilize low-resolution models of proteins created by structure prediction algorithms such as threading or $a b$ initio folding to predict the conformation of receptor-small ligand complexes. In our approach, using only approximate, discretized models of both molecules, we search for the steric and quasi-chemical complementarity between a ligand and the receptor molecules. This averaging procedure allows for the compensation of numerous structural inaccuracies resulting from the theoretical predictions of the receptor structure. The best relative orientation of these two models is obtained by an exhaustive scan over the rigid body's six-dimensional translational and rotational degrees of freedom. The search method is based on a real space grid-searching algorithm, unlike docking methods based on the fast Fourier Transform algorithm. We have applied this algorithm to rebuild structures of several complexes available in the Protein Data Bank. The structures of the receptors are produced by means of our threading algorithm PROSPECTOR, subsequently refined, and then utilized in the docking experiment. In many cases, not only is the localization of the binding site on the receptor surface correctly identified, but the proper orientation of the bounded ligand is also reasonably well reproduced within the level of accuracy of the modeled receptor itself.
\end{abstract}

(C) 2002 John Wiley \& Sons, Inc. J Comput Chem 23: 189-197, 2002

Key words: ligand docking; protein structure prediction; low-resolution protein models; cocrystallized complexes; induced fit

\section{Introduction}

One of the important, if not the most important, elements of protein function is the protein's ability to interact with and bind various ligands. This ability is closely related to the three-dimensional structure of the protein. Although the number of known primary sequences of proteins grows rapidly, their quaternary structures usually remain unknown due to the relatively difficult and timeconsuming procedure of experimental structure determination. Recently, the quality of theoretical structure prediction methods has been greatly improved, and sometimes results in structures whose quality is similar to low-resolution experimental structures. ${ }^{1,2}$ Thus, there is a clear need for a docking procedure that will be able to utilize these theoretical models of proteins for the prediction of conformations of receptor-small ligand complexes. Such an approach might also be helpful in rebuilding the quaternary structures of multimeric proteins when the structures of particular subunits of the protein are also theoretically predicted.

There are many approaches to the docking problem, and many algorithms developed by various groups have been devoted to this problem. ${ }^{3-7}$ Many approaches consider both the ligand and the re- ceptor to be rigid, ${ }^{8-10}$ while still others try to deal with the ligands and, to some extent, the receptor's flexibility. $5,11,12$ There are also various methodologies used for scoring the quality of the resulting complexes. Some implementations use simple geometric criteria such as surface and shape complementarity to define the binding site, ${ }^{13,14}$ while others use some type of potential energy function to distinguish between good and bad solutions. ${ }^{15-17}$

Shape complementarity plays an important role in proteinprotein interactions, ${ }^{18,19}$ and various techniques have proven to be efficient tools for generating near-native conformations of complexes, even from unbound components. ${ }^{20}$ Recently, methods based on correlation functions have become very popular. ${ }^{7,} 8,21,22$ In these algorithms, the structures of the molecules to be docked are first discretized by projecting them onto a three-dimensional grid and then the value of a correlation function that accounts for the shape complementarity of these two discrete representations is calculated in a search over the six-dimensional rigid body degrees of

Correspondence to: J. Skolnick; e-mail: skolnick@danforthcenter.org Contract/grant sponsor: NIH; contract/grant number: RR12255 
freedom. This search can be very efficiently performed by applying a Fourier transformation. An additional advantage of algorithms that utilize a correlation function is their ability to accept, to some extent, inaccuracies in the models by computationally changing the grid size. This type of protocol should make docking calculations for low-resolution structures possible. ${ }^{22-25}$

Although algorithms for the successful docking of lowresolution structures of pairs of proteins have recently improved, ${ }^{16,26}$ the problem of docking small ligands to such receptors is unexplored. In this article, we approach the problem of docking small ligands to inaccurate receptor structures by searching for both the steric and chemical complementarity between the ligand and the receptor molecule. Because our main focus is on docking to low-resolution structures that are in most cases the results of theoretical predictions, we use only approximate, discretized models of both the ligand and its protein receptor. Previously, it was shown that by averaging the structural details and by smoothing the potential energy surface, it is possible to drive the ligand towards the real binding site; thus avoiding, in many cases, the local minima problem. ${ }^{21,27}$ In our case it also turns out that this averaging procedure allows for the compensation of numerous structural errors resulting from theoretical predictions of the receptor's tertiary structure.

We have applied our new algorithm to rebuild structures of several complexes available in the Protein Data Bank. The structures of these receptors were first predicted from our threading algorithm, ${ }^{28}$ refined using our generalized comparative model protocol, ${ }^{29}$ and then utilized in the docking experiments. In many cases, not only has the localization of the binding site on the receptor surface been correctly identified, but the proper orientation of the bound ligand was reasonably restored, well within the level of accuracy of the modeled receptor.

\section{Methods}

Our docking procedure is a grid-based, complete search over the six-dimensional space defined by the rigid body translation of the ligand in three dimensions and its rotation over three Euler angles. No additional information regarding the binding site is required. Before performing the actual docking procedure, we assign "properties" to every atom of the ligand molecule. This assignment just defines to which of the 19 predefined chemical groups (see Table 1) the particular atom of the ligand belongs.

In the first step of the algorithm, both the receptor and the ligand are discretized by projecting them onto a uniform cubic lattice of grid size $2 \AA$. The projection of the ligand onto the grid is performed such that if the distance of the centroid of any cell to an atom of the ligand is smaller than the size of the cell, then this cell is marked by the property of the group of which this atom is a member.

The structure of the receptor is projected in a slightly different manner. This process is divided into two stages (see Fig. 1). In the first stage, all lattice cells that lie within the distance of double the cell size from any atom of an amino acid side chain or its alpha carbon are marked as having the property of that particular amino acid. Backbone atoms, excluding the alpha carbons, are projected as an additional, virtual, amino acid type. At the same time, all the
Table 1. Fragments and Functional Groups Used in the Definition of the Ligand-Amino Acid Statistical Potential.

\begin{tabular}{cll}
\hline Number & \multicolumn{1}{c}{ Symbol } & \multicolumn{1}{c}{ Group Description } \\
\hline 1 & $-\mathrm{COOH}$ & carboxylic acid \\
2 & $-\mathrm{CONH}-$ & amide \\
3 & $-\mathrm{NH}_{2}$ & amine \\
4 & $-\mathrm{NH}_{2}$ & amine by multiple bond \\
5 & $-\mathrm{OH}$ & hydroxyl \\
6 & $-\mathrm{SH}$ & thiol \\
7 & $-\mathrm{Ph}$ & phenyl \\
8 & $-\mathrm{C}-\mathrm{C}-$ & chain of aliphatic carbons \\
9 & $-\mathrm{C}=\mathrm{C}-$ or $-\mathrm{C} \equiv \mathrm{C}-$ & fragment of chain with \\
& & multiple $\mathrm{CC}$ bonds \\
10 & $-\mathrm{NHC}\left(\mathrm{NH} \mathrm{H}_{2}\right) \mathrm{NH}$ & guanidinium \\
11 & & heterocyclic ring \\
12 & $-\mathrm{C}-\mathrm{S}-\mathrm{C}-$ & thioether \\
13 & $-\mathrm{C}-\mathrm{O}-\mathrm{C}-$ & ether \\
14 & $>\mathrm{C}=\mathrm{O}$ & carbonyl \\
15 & $-\mathrm{SO}_{2}-$ & sulfone \\
16 & $-\mathrm{SO}_{3} \mathrm{H}$ & sulfonic acid \\
17 & $-\mathrm{PO}_{4}-$ & phosphate \\
18 & $-\mathrm{NO}_{2}$ & nitro group \\
19 & $-\mathrm{X}$ & halogene $(\mathrm{F}, \mathrm{Cl}, \mathrm{Br})$ \\
\hline
\end{tabular}

lattice cells projected by side chain atoms are marked as the receptor's "SHELL," while lattice cells projected by backbone atoms are marked as receptor "COAT" cells. In the second stage, the projection of the receptor is repeated. This time the particular cell is marked as belonging to the attractive COAT of the receptor if its centroid is located within the distance of one cell size from the side chain atom of any amino acid. A cell is marked as belonging to the repulsive CORE of the receptor molecule if it is located within the distance of one cell size from any protein backbone atom, excluding the alpha carbons. In the case of the receptor molecule, not only are the exact positions of the side chain atoms used for the projection, but the positions of these atoms resulting from all the rotameric states of every residue are also used.

After both molecules are discretized, their best relative orientation or, to be more precise, the best relative orientation of their discretized images, is obtained by an exhaustive search over the entire grid space, which is conducted by moving the set of cells representing the ligand molecule. This movement is performed using a one grid cell step. When the scanning of the grid by the ligand cells' translation is complete, the ligand molecule is rotated by one of the Euler angles. Then, its new orientation is again projected onto the lattice, and the whole search process starts over again from the beginning. The projection, grid scanning, and ligand rotation steps are repeated until the entire sixdimensional relative translation-orientation space is exhaustively searched.

During the search, each position of the discretized ligand molecule is scored according to its steric complementarity with the particular area of the receptor grid calculated for the value of the correlation function. Additionally, the energy of the interaction is calculated according to the scoring by the knowledge-based pairwise potential. 


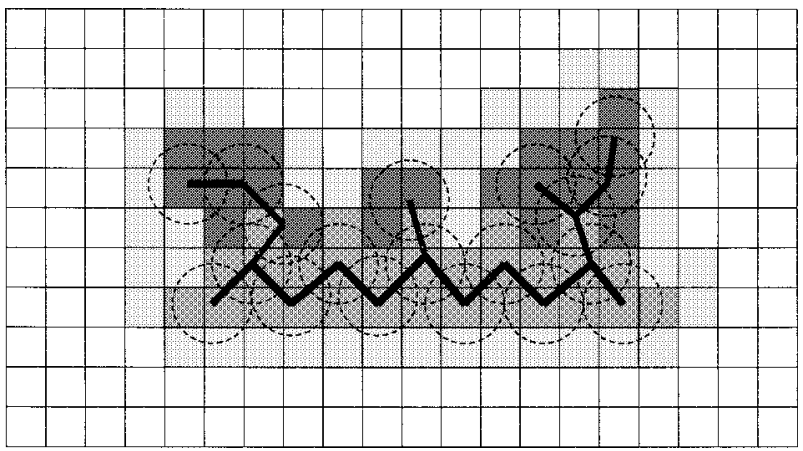

Figure 1. The projection of the receptor residues to the cubic lattice. Light gray represents the SHELL cubes, medium gray represents the CORE cubes, and dark gray represents the COAT cubes.

The value of the steric complementarity is evaluated by means of a simple correlation function:

$$
S_{\alpha, \beta, \gamma}=\sum_{l=1}^{N} \sum_{m=1}^{N} \sum_{n=1}^{N} a_{l, m, n} * b_{l+\alpha, m+\beta, n+\gamma}
$$

where:

$$
a_{l, m, n}=\left\{\begin{array}{cl}
-5 & \text { CORE } \\
0.5 & \text { COAT } \\
0.5 * n & \text { SHELL } \\
0 & \text { environament }
\end{array} \quad b=\left\{\begin{array}{l}
1 \\
0
\end{array}\right.\right.
$$

In this representation, the only repulsive part of the interaction comes from the ligand cubes overlapping with CORE receptor cubes, i.e., those cubes, which are the discretized representation of the receptor-backbone atoms. The cubes that represent the receptor side chains contribute an attractive part in the interaction score. In particular, cubes in the SHELL of the molecule are attractive with the strength of this interaction proportional to $n$, the number of the receptor amino acids projected into this cube.

Because docking calculations based only on steric complementarity (especially when dealing with small ligand molecules) usually lead to incorrect results due to a large number of false positives, we additionally use a pairwise statistical potential to score the resulting complexes according to their quasichemical complementarity. The specific part of the interaction in our scoring function is based on a pairwise statistical potential built on the basis of over 300 known structures of various complexes available in the Protein Data Bank (PDB). ${ }^{30}$ The structures used to derive the statistical potential were selected from the whole PDB database according to the following rule: only those structures with ligands containing at least above 5 heavy atoms were chosen. Those structures with any other ligands (listed as HET records in the PDB file) within the range of $8 \AA$ from the chosen one were rejected. All sequences with a sequence identity above $50 \%$ to any other sequence in the set were also removed from the database. Two structures with an identity above $50 \%$ were accepted into the training set of protein structures only if their ligands had different PDB three-letter code names and, more importantly, if their sizes differed by at least five heavy atoms. Extrinsic to the training set, a testing set of 20 com- plexes was selected according to similar criteria, except that none of the structures included in the testing set was allowed to have a sequence identity higher than $20 \%$ to any of the structures already included in the above-mentioned training set.

To build the potential, we defined 19 functional groups used to decompose the structure of the ligand into quasichemical building blocks. The groups used in our analysis are listed in Table 1. The parameters of our potential were obtained by a statistical analysis of the training set of complexes described above, and then applying eq. (2).

$$
E_{i, j}=-\ln \frac{n_{i, j}}{N \cdot x_{i} \cdot x_{j}}
$$

where $n_{i, j}$ is the number of observed contacts of the functional group $i$ with amino acid of type $j, x_{i}$, and $x_{j}$ are the mol fractions of groups $i$ and amino acids $j$, respectively, and $N$ is the total number of contacts in the database. In our approach, a contact between $i$ and $j$ occurs when the ligand cell marked with property $i$ overlaps with the receptor cell marked with the property of the amino acid $j$. Obviously, this potential depends on the lattice cell size. In all of our calculations, we used a fixed grid size of $2 \AA$. This was arrived at on the basis of several computational experiments, which showed that the best results are obtained with this grid size.

The specific interaction score was calculated according to eqs. (3) and (4), respectively, as

$$
\begin{aligned}
p & =\sum_{i=1}^{21} \sum_{j=1}^{19} E_{i, j} * n_{i} \\
P_{\alpha, \beta, \gamma} & =\sum_{l=1}^{N} \sum_{m=1}^{N} \sum_{n=1}^{N} p_{l+\alpha, m+\beta, n+\gamma}
\end{aligned}
$$

The final docking score of the particular complex was calculated by means of the linear combination of both the steric and the potential terms according to eq. (5).

$$
\mathrm{DS}=\operatorname{corr} * S+P
$$

where corr is a correction term that depends on the size of the ligand molecule, $S$ is the value of the steric match term calculated by eq. (1), and $P$ is the value of the potential specific interaction term calculated by eq. (4). The correction term is used to ensure that both $P$ and $S$ have a similar influence on the value of the docking score DS, and it is calculated by eq. (6), in which $n$ is the number of atoms in the ligand molecule. The coefficients of this equation are estimated as the least-square approximations of the values of $S$ and $P$ on the basis of calculations for the set of native complexes.

$$
\text { corr }=\frac{4.23 * n+40.08}{1.32 * n+22.20}
$$

After the entire six-dimensional space is searched, all of the solutions are scored according to eq. (5) and then sorted. 


\section{Results and Discussion}

We used a program written on the basis of the above algorithm to dock some small ligands to the structures of the receptors available from the PDB, as both are complexes with these ligands as well as with a number of theoretically predicted models of the receptors. The complete list of ligands and receptors used can be found on our Web page http://bioinformatics.danforthcenter.org/. To be able to easily verify the docking results in the test cases, when the correct geometry of the complex was known, we need a measure that scores the quality of the predicted complexes relative to the native ones. We decided to use (as a quality indicator) the percentage of the native contacts $(\mathrm{NC})$ from the original native complex that were preserved in the predicted complex as well. We count a contact as being native if ligand atom number $i$ is in contact with receptor residue number $j$ in both the predicted and the native complexes. Additionally, we also used the percentage of nonspecific contacts (nsNC) as a measure of the success of the localization of the binding site residues. The percentage of nonspecific contacts is defined as the fraction of residues that are in contact with the ligand in the predicted complex relative to the fraction of the residues that are in contact with the ligand in the native structure.

First, we tested our algorithm on the database of 318 complexes that we used to generate our statistical potential (see Table 2). We repeated these calculations with only the steric complementarity term used for scoring, then with only the statistical potential term, and finally with the complete term used for scoring the complexes. In all runs, a grid size of $2 \AA$ was used, and the ligand rotation step was set to $20^{\circ}$. All calculations were performed on a $733-\mathrm{MHz}$ Pentium III cluster. The average job took about 5-10 min on 20 processors.

The results of these calculations are presented in Figure 2. As one can easily see, the scoring function that combines both the steric complementarity term and the qasichemical potential performs better than either alone. This combined function allows us to predict the most complexes with a particular number of preserved native contacts. However, a closer analysis of the curves showing the results for the steric only and potential only docking reveals an interesting relationship. Scoring by steric complementarity only gives a few complexes with very good quality (the percentage of preserved native contacts is above 80-90\%), but it is able to predict about 200 structures out of 318 with at least one native contact. Although the best quality predictions made by means of the potential-only scoring have fewer, mainly only $50-60 \%$, of their native contacts preserved, the percentage of the predicted contacts drops more slowly than in the previous case to give 230 complexes overall, with at least one native contact. This result clearly indicates that the binding site and its surroundings maintain specificity towards the ligand even in this low-resolution representation. On the other hand, the number of correctly predicted complexes obtained by scoring only with the steric complementarity term is also surprisingly high, especially when one keeps in mind that a fuzzy representation of the receptor side-chain positions is used in the calculations, and the ligands are relatively small.

The results using the combined set of terms, rank ordered by decreasing quality of the fraction of native ligand-receptor contacts that are correctly predicted, is summarized in Table 2 . The fraction of correctly predicted contacts ranges from 87 to $0 \%$.

Obviously these calculations do not say much about the real predictive power of our algorithm. To get a more reliable verification of its performance, we repeated the docking calculations for the smaller database of 20 test complexes, but this time with the additional restriction that not only were none of these structures present in the training set of complexes, but also that none of these structures had a sequence identity higher than $20 \%$ to any of the structures from this set. Table 3 a shows the set of testing proteins along with their corresponding ligand. As shown in Table 3b, in this case, 11 predicted complexes had $20 \%$ or more of the specific native contacts preserved, and 13 of them had at least $10 \%$ of the specific contacts preserved. This looks very promising in terms of the predictive power of the algorithm.

To test our algorithm under conditions closer to real-life problems when the geometry of the binding site differs sometimes significantly from the geometry in the cocrystallized complex, we performed additional docking calculations on a few examples of the ligands and receptors that were crystallized separately and whose structures are available in the PDB in both forms. Because, in most cases, the conformation of the receptor side chains readjust to the ligand only upon binding (the alpha carbon root mean square derivative, RMSD, for the receptors crystallized in the free form and the ones that cocrystallized with the ligands in most cases are below $1 \AA$ ) and because, in our model, the crystallographic side chain positions are not used explicitly for docking, not surprisingly we did not notice any significant differences in complexes obtained by redocking ligands to their cocrystallized receptors and the same complexes obtained after docking these ligands to the apropriate receptors crystallized in the free form.

Although in most cases the ligand-binding process involves only small side-chain rearrangements among the binding pocket residues, it may sometimes induce a wide range of the structural changes in a large part of the protein, including the hinge movements of the entire receptor subdomains. In our selected subset of the PDB, we found a few structures of cocrystallized complexes with the receptors also crystallized separately where the RMSD differences of the alpha-carbon positions between both the free and ligand-bound forms of the protein were even above $7 \AA$. We applied our algorithm to dock the ligands found in the cocrystallized forms to the free forms of these receptors. An example of a protein that shows significant ligand-induced domain movements is the maltodextrin binding protein. ${ }^{31}$ The structure of this protein is available in the PDB in both the free form (1omp) as well as in the form of complexes with various ligands (1anf, $3 \mathrm{mbp}$ ). In the free form, the binding site is open and accessible to the water molecules (Fig. 3a).

Upon ligand binding, this protein undergoes a hinge-bending and a twisting kind of motion between its two domains, so that, once bound, the ligand is closed inside the binding pocket (Fig. 3b). The RMSD of the alpha-carbon positions between these two forms of the receptor is about $3.7 \AA$; however, most of the differences are concentrated in the area of the binding site. The subdomains themselves behave, during ligand binding, almost as rigid bodies, and their internal geometries do not change much. Despite these structural differences, the docking of maltose (the ligand molecule from the 1anf structure) as well as maltotriose (the ligand molecule 
Table 2. The List of All Training PDB Structures and the Percentage of Preserved Native Contacts.

\begin{tabular}{|c|c|c|c|c|c|c|c|c|}
\hline No. & PDB ID & $\mathrm{NC}^{\mathrm{a}}$ & No. & PDB ID & $\mathrm{NC}^{\mathrm{a}}$ & No. & PDB ID & $\mathrm{NC}^{\mathrm{a}}$ \\
\hline 1 & 1rom & 87 & 58 & $1 \mathrm{bj} 9$ & 33 & 115 & $1 \mathrm{rms}$ & 17 \\
\hline 2 & 1 ars & 86 & 59 & $7 \mathrm{gch}$ & 32 & 116 & $2 \mathrm{cbs}$ & 16 \\
\hline 3 & $1 \mathrm{c} 24$ & 85 & 60 & $1 \mathrm{fkh}$ & 32 & 117 & $1 q c p$ & 16 \\
\hline 4 & $1 \mathrm{dt} 1$ & 78 & 61 & $1 \mathrm{clh}$ & 32 & 118 & $1 \mathrm{ck} 6$ & 16 \\
\hline 5 & $1 \mathrm{spa}$ & 75 & 62 & $1 \mathrm{db} 1$ & 32 & 119 & 1ayw & 16 \\
\hline 6 & $1 \mathrm{icm}$ & 75 & 63 & $2 \mathrm{hmb}$ & 32 & 120 & $1 b g q$ & 16 \\
\hline 7 & $1 \mathrm{ddt}$ & 65 & 64 & lbep & 31 & 121 & $1 \mathrm{qti}$ & 16 \\
\hline 8 & 1icn & 63 & 65 & 1 pax & 30 & 122 & 1au3 & 16 \\
\hline 9 & $1 \mathrm{map}$ & 62 & 66 & $1 \mathrm{pbk}$ & 29 & 123 & $1 \mathrm{hvq}$ & 16 \\
\hline 10 & $1 \operatorname{co6}$ & 62 & 67 & 3pyp & 29 & 124 & $2 \mathrm{nlr}$ & 16 \\
\hline 11 & $451 \mathrm{c}$ & 61 & 68 & laba & 29 & 125 & $1 \mathrm{ci} 3$ & 16 \\
\hline 12 & $1 \mathrm{c} 22$ & 60 & 69 & 3 pax & 29 & 126 & $1 \mathrm{~d} 4 \mathrm{o}$ & 16 \\
\hline 13 & $2 \mathrm{cmd}$ & 58 & 70 & $1 \mathrm{cxc}$ & 27 & 127 & $1 \mathrm{hlb}$ & 16 \\
\hline 14 & $1 \mathrm{maq}$ & 57 & 71 & lbek & 27 & 128 & $2 y p n$ & 16 \\
\hline 15 & 1iol & 57 & 72 & $1 \mathrm{drh}$ & 27 & 129 & $1 \mathrm{bb} 6$ & 15 \\
\hline 16 & livr & 57 & 73 & 1 cyo & 26 & 130 & $1 \mathrm{gsq}$ & 15 \\
\hline 17 & $5 y a s$ & 57 & 74 & $1 \mathrm{c} 11$ & 26 & 131 & 1lce & 15 \\
\hline 18 & $1 \mathrm{akc}$ & 57 & 75 & $2 \mathrm{hbg}$ & 26 & 132 & 1oyc & 15 \\
\hline 19 & 1aod & 55 & 76 & $5 \operatorname{tln}$ & 26 & 133 & 2ack & 15 \\
\hline 20 & $1 \mathrm{bxm}$ & 54 & 77 & 5 cyt & 25 & 134 & $1 \mathrm{akb}$ & 15 \\
\hline 21 & $1 \mathrm{hcz}$ & 54 & 78 & $1 \mathrm{myt}$ & 25 & 135 & 4 pax & 15 \\
\hline 22 & $1 \mathrm{zsb}$ & 52 & 79 & $1 \mathrm{~b} 8 \mathrm{o}$ & 25 & 136 & $1 \mathrm{cr} 1$ & 15 \\
\hline 23 & $3 \mathrm{c} 2 \mathrm{c}$ & 52 & 80 & $4 \mathrm{lbd}$ & 25 & 137 & 1eno & 15 \\
\hline 24 & 2 dri & 51 & 81 & 1oce & 25 & 138 & $1 \mathrm{cbs}$ & 15 \\
\hline 25 & $1 \mathrm{dtp}$ & 51 & 82 & $2 \mathrm{~mm} 1$ & 25 & 139 & $1 \mathrm{fkl}$ & 15 \\
\hline 26 & $10 x p$ & 50 & 83 & 1 bvd & 24 & 140 & 1ayv & 15 \\
\hline 27 & $5 \mathrm{rhn}$ & 50 & 84 & 3dhe & 24 & 141 & 1ojt & 14 \\
\hline 28 & $1 \mathrm{cot}$ & 48 & 85 & $1 \mathrm{a} 53$ & 24 & 142 & $1 \mathrm{fem}$ & 14 \\
\hline 29 & $1 \mathrm{ylv}$ & 48 & 86 & $1 \mathrm{bb} 7$ & 24 & 143 & $1 \mathrm{fen}$ & 14 \\
\hline 30 & 1zid & 47 & 87 & $3 \mathrm{lbd}$ & 23 & 144 & $1 \mathrm{ra} 9$ & 13 \\
\hline 31 & $3 \mathrm{rhn}$ & 45 & 88 & 1yet & 23 & 145 & $3 \mathrm{cbs}$ & 13 \\
\hline 32 & 5 bu 4 & 44 & 89 & $2 \mathrm{fcr}$ & 23 & 146 & 1ndh & 13 \\
\hline 33 & $1 \mathrm{ctj}$ & 44 & 90 & $1 \mathrm{flp}$ & 23 & 147 & $1 \operatorname{arc}$ & 13 \\
\hline 34 & 3ert & 43 & 91 & lbgo & 22 & 148 & $1 \mathrm{mnp}$ & 13 \\
\hline 35 & $1 \mathrm{rpj}$ & 43 & 92 & $1 \mathrm{~d} 7 \mathrm{r}$ & 22 & 149 & $7 \mathrm{taa}$ & 12 \\
\hline 36 & 2dap & 41 & 93 & 5fit & 22 & 150 & $1 \mathrm{shv}$ & 12 \\
\hline 37 & 1lih & 41 & 94 & $1 \mathrm{htp}$ & 22 & 151 & $4 \mathrm{mbp}$ & 12 \\
\hline 38 & $1 d 7 v$ & 40 & 95 & $1 \mathrm{drv}$ & 22 & 152 & $1 \mathrm{cef}$ & 12 \\
\hline 39 & $1 \mathrm{c} 75$ & 40 & 96 & 5eat & 21 & 153 & $2 \mathrm{fam}$ & 11 \\
\hline 40 & $1 \mathrm{drm}$ & 39 & 97 & $1 \mathrm{~b} 56$ & 21 & 154 & $1 \mathrm{rg} 7$ & 11 \\
\hline 41 & $6 \mathrm{qch}$ & 38 & 98 & 6nul & 21 & 155 & $1 \mathrm{cr} 2$ & 11 \\
\hline 42 & $1 \mathrm{a} 3 \mathrm{k}$ & 38 & 99 & $1 \mathrm{c} 9 \mathrm{e}$ & 20 & 156 & $1 \mathrm{bp} 4$ & 11 \\
\hline 43 & $1 \mathrm{kpf}$ & 38 & 100 & $1 \mathrm{a} 4 \mathrm{~h}$ & 20 & 157 & $1 \mathrm{qs} 2$ & 11 \\
\hline 44 & $21 \mathrm{hb}$ & 38 & 101 & $1 \mathrm{fkd}$ & 20 & 158 & $1 \mathrm{jdd}$ & 11 \\
\hline 45 & $1 \mathrm{ceq}$ & 37 & 102 & $3 \mathrm{a} 3 \mathrm{~h}$ & 20 & 159 & $2 \mathrm{fke}$ & 11 \\
\hline 46 & $1 \mathrm{ptg}$ & 37 & 103 & 1 bso & 20 & 160 & $1 \mathrm{~d} 06$ & 11 \\
\hline 47 & $1 \mathrm{mrk}$ & 37 & 104 & 1nje & 20 & 161 & 1blh & 11 \\
\hline 48 & 7odc & 36 & 105 & $7 \mathrm{ccp}$ & 20 & 162 & $5 \mathrm{f} \times 2$ & 10 \\
\hline 49 & $1 \mathrm{fkg}$ & 36 & 106 & $1 \mathrm{dmb}$ & 20 & 163 & $1 \mathrm{vzc}$ & 10 \\
\hline 50 & $1 \mathrm{cpq}$ & 36 & 107 & $1 \mathrm{cxy}$ & 19 & 164 & $1 \mathrm{aec}$ & 10 \\
\hline 51 & $1 \mathrm{fsz}$ & 35 & 108 & $1 \mathrm{au} 2$ & 19 & 165 & $1 \mathrm{~b} 02$ & 10 \\
\hline 52 & $1 \mathrm{mpd}$ & 35 & 109 & 1gne & 19 & 166 & $1 \mathrm{eco}$ & 10 \\
\hline 53 & $1 \mathrm{pmt}$ & 35 & 110 & $2 \mathrm{sim}$ & 18 & 167 & $1 \mathrm{~b} 8 \mathrm{n}$ & 10 \\
\hline 54 & 11lo & 34 & 111 & $1 \mathrm{fhe}$ & 17 & 168 & 1aim & 9 \\
\hline 55 & $1 \mathrm{~b} 9 \mathrm{i}$ & 34 & 112 & 1tyn & 17 & 169 & 1bo8 & 9 \\
\hline 56 & lngh & 34 & 113 & $1 \mathrm{qsr}$ & 17 & 170 & $2 \ln 5$ & 9 \\
\hline 57 & $1 \mathrm{qkq}$ & 33 & 114 & $1 \mathrm{cgo}$ & 17 & 171 & $1 \mathrm{~b} 9 \mathrm{~h}$ & 9 \\
\hline
\end{tabular}


Table 2. (Continued)

\begin{tabular}{|c|c|c|c|c|c|c|c|c|}
\hline No. & PDB ID & $\mathrm{NC}^{\mathrm{a}}$ & No. & PDB ID & $\mathrm{NC}^{\mathrm{a}}$ & No. & PDB ID & $\mathrm{NC}^{\mathrm{a}}$ \\
\hline 172 & 1 tsl & 9 & 221 & $1 b 9 t$ & 0 & 270 & $1 \mathrm{obt}$ & 0 \\
\hline 173 & $2 p a x$ & 9 & 222 & $1 \mathrm{qpk}$ & 0 & 271 & 1rbn & 0 \\
\hline 174 & $1 \mathrm{~b} 0 \mathrm{f}$ & 8 & 223 & $1 \mathrm{drw}$ & 0 & 272 & 1upj & 0 \\
\hline 175 & $2 \mathrm{cbr}$ & 8 & 224 & $1 \mathrm{a} 39$ & 0 & 273 & $2 \mathrm{tdm}$ & 0 \\
\hline 176 & 1ayu & 7 & 225 & $1 \mathrm{axb}$ & 0 & 274 & $1 \mathrm{dgy}$ & 0 \\
\hline 177 & $1 \mathrm{hbp}$ & 7 & 226 & $1 \operatorname{cg} 6$ & 0 & 275 & $1 \mathrm{~b} 39$ & 0 \\
\hline 178 & 1bdu & 7 & 227 & linv & 0 & 276 & $1 \mathrm{bsj}$ & 0 \\
\hline 179 & 1lif & 7 & 228 & $1 \mathrm{bzc}$ & 0 & 277 & $1 \mathrm{pjc}$ & 0 \\
\hline 180 & $1 \mathrm{rx} 7$ & 7 & 229 & $1 \mathrm{bzj}$ & 0 & 278 & $3 \operatorname{cox}$ & 0 \\
\hline 181 & 1au0 & 6 & 230 & 1 cy6 & 0 & 279 & $1 \mathrm{~b} 8 \mathrm{v}$ & 0 \\
\hline 182 & $1 \mathrm{hnl}$ & 6 & 231 & $1 \mathrm{~b} 0 \mathrm{e}$ & 0 & 280 & 3eng & 0 \\
\hline 183 & $1 \mathrm{hna}$ & 6 & 232 & $1 \mathrm{jdx}$ & 0 & 281 & 1qan & 0 \\
\hline 184 & $4 \mathrm{tmk}$ & 6 & 233 & $1 \mathrm{bdb}$ & 0 & 282 & $3 \mathrm{jdw}$ & 0 \\
\hline 185 & $1 \mathrm{dad}$ & 6 & 234 & $1 \mathrm{~b} 1 \mathrm{c}$ & 0 & 283 & $1 \mathrm{qg} 2$ & 0 \\
\hline 186 & $1 \mathrm{a} 26$ & 6 & 235 & 1 by 2 & 0 & 284 & $1 \mathrm{qgf}$ & 0 \\
\hline 187 & $2 \mathrm{dhn}$ & 6 & 236 & $1 \mathrm{a} 27$ & 0 & 285 & $1 \mathrm{du} 7$ & 0 \\
\hline 188 & $1 \mathrm{erb}$ & 6 & 237 & 1may & 0 & 286 & $3 \mathrm{rab}$ & 0 \\
\hline 189 & 4 fiv & 6 & 238 & 1 dru & 0 & 287 & 1qra & 0 \\
\hline 190 & $1 \mathrm{fel}$ & 5 & 239 & $1 \mathrm{zfj}$ & 0 & 288 & $1 \mathrm{enu}$ & 0 \\
\hline 191 & $1 \mathrm{hmr}$ & 5 & 240 & $1 \mathrm{mrj}$ & 0 & 289 & $4 \mathrm{a} 3 \mathrm{~h}$ & 0 \\
\hline 192 & 1 vot & 5 & 241 & $1 \mathrm{dud}$ & 0 & 290 & $2 \operatorname{csn}$ & 0 \\
\hline 193 & 4rsk & 5 & 242 & $1 \mathrm{a} 5 \mathrm{w}$ & 0 & 291 & 1aj6 & 0 \\
\hline 194 & 1 br6 & 5 & 243 & $1 \mathrm{cgk}$ & 0 & 292 & 1eus & 0 \\
\hline 195 & 1adg & 5 & 244 & 2aim & 0 & 293 & $1 \mathrm{ama}$ & 0 \\
\hline 196 & 1lid & 4 & 245 & $1 \mathrm{aqm}$ & 0 & 294 & 1lsp & 0 \\
\hline 197 & $5 \mathrm{tmp}$ & 4 & 246 & $2 \mathrm{cah}$ & 0 & 295 & $1 \mathrm{amq}$ & 0 \\
\hline 198 & $1 \mathrm{rob}$ & 4 & 247 & $1 \mathrm{cip}$ & 0 & 296 & $2 \mathrm{enb}$ & 0 \\
\hline 199 & 1adf & 4 & 248 & $1 \mathrm{cjc}$ & 0 & 297 & $5 \mathrm{a} 3 \mathrm{~h}$ & 0 \\
\hline 200 & 1 trb & 3 & 249 & 1cet & 0 & 298 & $1 \mathrm{btn}$ & 0 \\
\hline 201 & $1 \mathrm{ofv}$ & 3 & 250 & $1 \operatorname{cg} 4$ & 0 & 299 & $1 \mathrm{rsm}$ & 0 \\
\hline 202 & $1 \mathrm{gr} 2$ & 3 & 251 & $2 \mathrm{a} 3 \mathrm{~h}$ & 0 & 300 & $1 \mathrm{rvd}$ & 0 \\
\hline 203 & $4 \mathrm{~cd} 2$ & 3 & 252 & $1 \mathrm{~b} 0 \mathrm{u}$ & 0 & 301 & $1 \mathrm{bvq}$ & 0 \\
\hline 204 & $1 \mathrm{fdr}$ & 3 & 253 & $1 \mathrm{cpt}$ & 0 & 302 & 1bws & 0 \\
\hline 205 & $1 \mathrm{bib}$ & 3 & 254 & 2ang & 0 & 303 & $1 \mathrm{frq}$ & 0 \\
\hline 206 & $1 c 9 w$ & 2 & 255 & $1 \mathrm{ctq}$ & 0 & 304 & $1 \mathrm{skj}$ & 0 \\
\hline 207 & $2 \mathrm{cnd}$ & 2 & 256 & 1aq7 & 0 & 305 & $1 \mathrm{fxs}$ & 0 \\
\hline 208 & $1 \mathrm{rpf}$ & 2 & 257 & 1iam & 0 & 306 & 1sth & 0 \\
\hline 209 & $1 \mathrm{tcs}$ & 2 & 258 & $1 \mathrm{cw} 7$ & 0 & 307 & $6 \mathrm{cts}$ & 0 \\
\hline 210 & $11 \mathrm{mc}$ & 2 & 259 & $1 \mathrm{mbt}$ & 0 & 308 & 6fiv & 0 \\
\hline 211 & $2 \mathrm{dpg}$ & 2 & 260 & 1 cy 4 & 0 & 309 & 1gym & 0 \\
\hline 212 & $1 \mathrm{rx} 5$ & 2 & 261 & $1 \mathrm{fmb}$ & 0 & 310 & $2 \mathrm{q} 21$ & 0 \\
\hline 213 & 1byg & 2 & 262 & $2 \mathrm{gnk}$ & 0 & 311 & 1uib & 0 \\
\hline 214 & $2 \mathrm{dpm}$ & 2 & 263 & $1 \mathrm{bx} 6$ & 0 & 312 & $1 \mathrm{hdr}$ & 0 \\
\hline 215 & 1ifu & 1 & 264 & $1 \mathrm{mtw}$ & 0 & 313 & $7 \mathrm{jdw}$ & 0 \\
\hline 216 & $1 \mathrm{enz}$ & 1 & 265 & $1 \mathrm{~d} 01$ & 0 & 314 & $1 \mathrm{vpt}$ & 0 \\
\hline 217 & $1 \mathrm{~b} 0 \mathrm{o}$ & 1 & 266 & $1 \mathrm{~d} 6 \mathrm{~h}$ & 0 & 315 & 1diw & 0 \\
\hline 218 & $1 \mathrm{a} 8 \mathrm{p}$ & 1 & 267 & $1 \mathrm{~d} 6 \mathrm{f}$ & 0 & 316 & 8est & 0 \\
\hline 219 & $1 \mathrm{cgz}$ & 1 & 268 & $2 \mathrm{ncd}$ & 0 & 317 & 9est & 0 \\
\hline 220 & $1 \mathrm{dih}$ & 1 & 269 & 1 nox & 0 & 318 & 9icd & 0 \\
\hline
\end{tabular}

${ }^{\mathrm{a}} \mathrm{NC}$ is the fraction of preserved native contacts.

from the $3 \mathrm{mbp}$ structure) to the free receptor (1omp) resulted in the structure of a complex with the ligands positioned in the correct area of the binding site (Fig. 3).

Another group of proteins that are known to undergo significant conformational changes upon binding are kinases. ${ }^{32}$ Figure 4 shows adenylate kinase complexed with its inhibitor (Fig. 4a with the complex's PDB code 1ake) and the same receptor crystallized in the free form (PDB code 4ake), but complexed with the inhibitor by means of our docking program (Fig. 4b). In this case, the RMSD between the receptor in its free (open) and the ligand- 


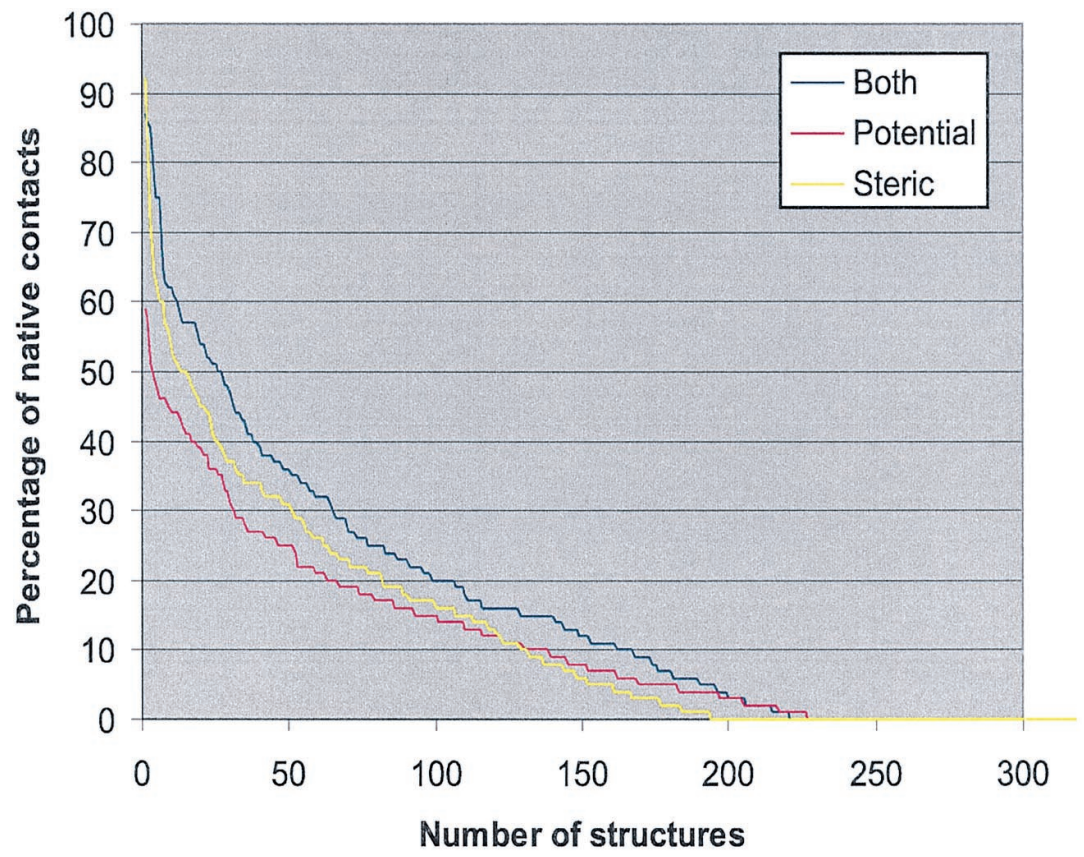

Figure 2. The number of complexes predicted by means of various scoring functions with the particular number of specific native contacts preserved.

bound (closed) form is $7.1 \AA$. Although the geometry of the binding site as well as the geometry of a large part of the protein is completely different in the free and in the complexed form of the kinase, even in this case our program was able to correctly identify the

Table 3a. The List of Ligands Used in the Nonhomologous Testing Set of PDB Complexes.

PDB

ID

1 af7

$1 \mathrm{~b} 3 \mathrm{n}$

$1 \mathrm{~b} 59$

$1 b j 4$

1 bym

1 cen

$1 \mathrm{dmw}$

$1 \mathrm{dve}$

$1 \mathrm{dvp}$

1 mai

$1 \mathrm{mdr}$

1npx

1nst

1oth

$1 \mathrm{qkp}$

1rne

lukd

$2 \mathrm{hmy}$

2izj

5 pnt
The Name of a Ligand as it Appears in the HETNAM (or HETSYN When Available) Records

S-Adenosyl-L-homocysteine

Cerulenin

Ovalicin

Pyridoxal-5' -phosphate

Glucose

Glucose

7,8-Dihydrobiopterin

Protoporphyrin IX containing FE

Citric acid

D-Myo-inositol-1,4,5-triphosphate

(S)-Atrolactic acid

Flavin-adenine dinucleotide

Adenosine- $3^{\prime} 5^{\prime}$-diphosphate

$\mathrm{N}$-(Phosphonoacetyl)-L-ornithine

Retinal

[[[3-(2-Methyl-propane-2-sulfonyl)-1-benzenyl]-2-propyl]carbonyl-histidyl]-amino-[Cyclohexylmethyl]-

[2-hydroxy-4-isopropyl]-pentan-5-oic acid butylamide

P1-(adenosine- $5^{\prime}$-P5-(uridine- $5^{\prime}$ ) pentaphosphate

S-Adenosylmethionine

Biotin

2-(N-Morpholino)-ethanesulfonic acid binding-site residues. When comparing residues in contact with the ligand molecule in the cocrystallized native complex with residues in contact in the complex obtained by means of our docking algorithm, in the predicted complex the docked inhibitor is in contact with a subset of residues that are also in contact with this ligand in the cocrystallized complex. Obviously, it is only a subset of these residues due to the significantly different geometry of the binding site in the open form of the receptor (Fig. 4b). However, the orientation of the bound inhibitor in the docked complex is similar to the one in the native cocrystallized complex.

Our main goal here was to develop an algorithm that would be able to dock smaller ligands to low-resolution, theoretically

Table 3b. Percentage of Preserved Specific and Nonspecific Native Contacts in the Docked Complexes for the Nonhomologous Testing Set.

\begin{tabular}{lccccc}
\hline PDB ID & $\mathrm{nNC}^{\mathrm{a}}(\%)$ & $\mathrm{NC}(\%)^{\mathrm{b}}$ & PDB ID & $\mathrm{nNC}^{\mathrm{a}}(\%)$ & $\mathrm{NC}(\%)^{\mathrm{b}}$ \\
\hline 1 oth & 89 & 87 & $2 \mathrm{izj}$ & 35 & 23 \\
1cen & 77 & 61 & $1 \mathrm{npx}$ & 55 & 18 \\
$1 \mathrm{dvp}$ & 80 & 47 & $1 \mathrm{nst}$ & 54 & 11 \\
1bj4 & 75 & 45 & $1 \mathrm{ukd}$ & 70 & 7 \\
1b59 & 79 & 41 & $1 \mathrm{af7}$ & 40 & 5 \\
1mai & 48 & 39 & $1 \mathrm{byc}$ & 47 & 3 \\
1qkp & 60 & 30 & $2 \mathrm{hmy}$ & 0 & 0 \\
$1 \mathrm{dmw}$ & 31 & 27 & $1 \mathrm{rne}$ & 3 & 0 \\
1b3n & 53 & 27 & $1 \mathrm{mdr}$ & 0 & 0 \\
$5 \mathrm{pnt}$ & 48 & 25 & $1 \mathrm{dve}$ & 21 & 0 \\
\hline
\end{tabular}

a $\mathrm{NC}$ is the fraction of preserved native contacts.

${ }^{b} \mathrm{nNC}$ is the percentage of nonspecific contacts, as defined in the Results and Discussion section. 

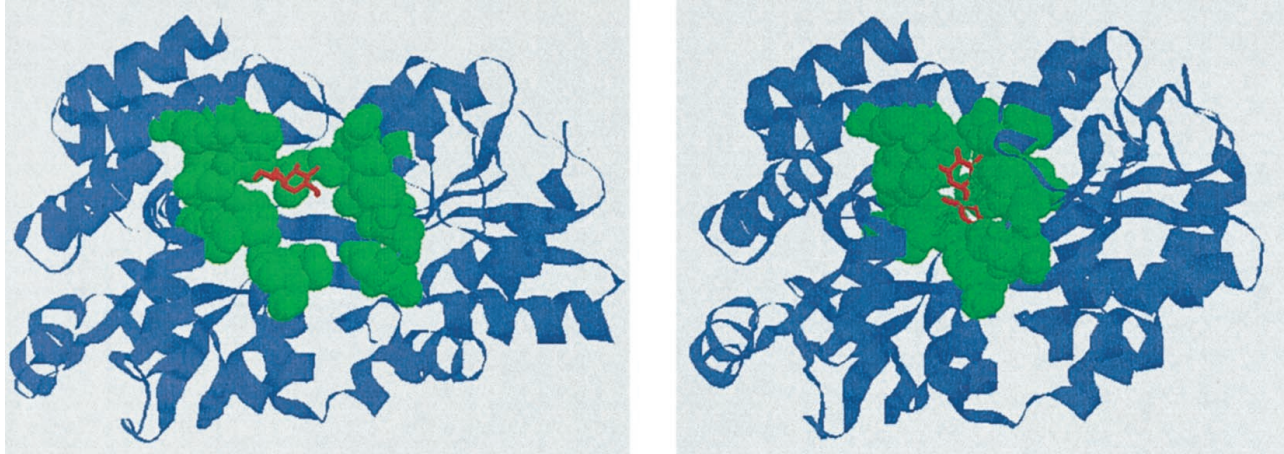

Figure 3. Maltodextrin binding protein. (a) The free (open) form with the ligand, maltose, docked. (b) The complexed (closed) form with the same ligand cocrystallized. Residues in contact with the ligand in the cocrystallized form are green.

predicted structures of receptors. Docking small molecules to the structures of receptors with significant differences from native is the real challenge. To test the efficiency of our protein structure prediction algorithms, we used a set of standard benchmarks, including the Fischer Database. ${ }^{33}$ Taking advantage of the fact that some of the structures present in the Fischer Database ${ }^{34}$ are also present in the PDB in the form of complexes with small ligands, we tested our docking procedure by trying to rebuild these complexes using our homology modeled structures of these receptors instead of the X-ray ones. The structures that were successfully modeled with reasonable accuracy and at the same time were available in the form of complexes with some small ligands have PDB ID codes as follows: $1 \mathrm{bbh}, 1 \mathrm{c} 2 \mathrm{r}, 1 \mathrm{mdc}, 2 \mathrm{cmd}$, 2 sar. The quality of these models is in the range of 3 to $6 \AA$ RMSD when compared to the appropriate PDB structures.

The results of the docking calculations are shown in Table 4. Even for the structures as far as almost $6 \AA$ from native, up to $47 \%$ of the specific native contacts are preserved. Only for the predicted $1 \mathrm{mdc}$ structure did docking fail to recognize the binding site. This structure is the fatty acid binding protein of a sulfate ion bound together with the ligand palmitic acid. The presence of this ion in the binding site was not taken into account in the docking experiment, but in this case it could be crucial for the correct ligand binding. ${ }^{35}$ All other complexes from this set were successfully rebuilt within the accuracy of the modeled receptor.
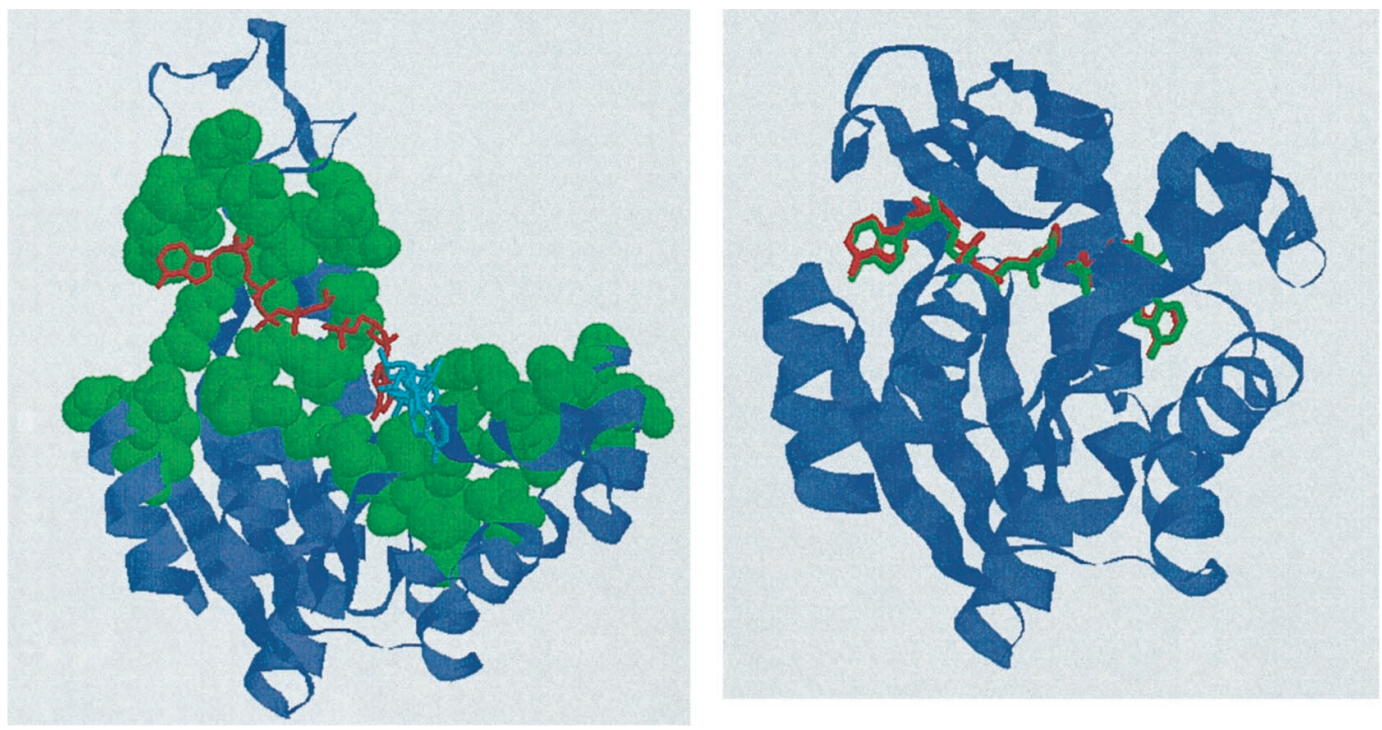

Figure 4. Adenylate kinase. (a) The free (open) form with the inhibitor docked by means of our algorithm. Two of the best scored complexes are shown. (b) The cocrystallized (closed) form. The original and redocked positions of the inhibitor are shown. Residues in contact with the ligand in the cocrystallized form are green. 
Table 4. Results of Docking to the Theoretically Predicted Receptor. ${ }^{\mathrm{a}}$

\begin{tabular}{lccc}
\hline PDB ID & RMSD $(\AA)$ & nsNC $(\%)$ & NC $(\%)$ \\
\hline 1bbhA & 3.16 & 75 & 47 \\
1c2rA & 4.94 & 71 & 30 \\
1mdc_- & 4.92 & 0 & 0 \\
2cmd_ & 5.57 & 74 & 47 \\
2 sarA & 5.99 & 42 & 59 \\
\hline
\end{tabular}

${ }^{a}$ RMSD of the modeled receptor from the experimental one. nsNC and NC are the percentage of nonspecific native contacts and percentage of specific native contacts, respectively.

\section{Discussion}

These results indicate that our docking routine is able to utilize structural information still present even in the low-resolution structures of receptors and to use this information to place small ligands in the binding site in the correct orientation. Local steric and physicochemical properties of the receptor binding sites are definitely responsible for the final locking of the ligand molecule in the correct position and orientation; however, these local preferences extend beyond the immediate neighborhood of the binding site itself. The general physicochemical properties of the receptor, mimicked here by our potential term, drive ligands toward the correct location on the receptor surface. It was shown previously ${ }^{22}$ that global structural features are an important factor in the first stages of protein-protein recognition. On the basis of our calculations, we conclude that, similarly, the global topology of the receptor is significant for binding small ligands as well, especially in the early stages. In the small ligand binding, we seem to be mimicking the first stage of the binding process, when the ligand is probing the surface of the receptor trying to find the areas with favorable interactions. At this point, the detailed structural features and high-resolution interactions like hydrogen bonding do not yet play an important role. It was shown that including such features, even in the docking of unbound molecules, does not influence the result. ${ }^{19}$ However, hydrogen bonds are obviously crucial in the next stage of binding, and including them in the scoring function significantly improves the results when restoring cocrystallized complexes. ${ }^{36}$

Although our algorithm is not perfect and, in some cases, fails to recognize the binding site for the particular receptor altogether, our results clearly indicate that in many cases it is possible to utilize even low-quality structures in successful docking experiments with small ligands. Obviously this procedure does not lead to a unique atomic-level solution. The resulting complexes must be further refined either by simple energy minimization or molecular dynamics calculations; however, when used in combination with other tools, our approach may prove to be very valuable for the genome-scale products of the ligand binding site.

\section{References}

1. Pillardy, J.; Czaplewski, C.; Liwo, A.; Lee, J.; Ripoll, D. R.; Kazmierkiewicz, R.; et al. Proc Natl Acad Sci USA 2001, 98, 2329.

2. Marchler-Bauer, A.; Bryant, S. H. Proteins 1999, 37, 218.

3. Gschwend, D. A.; Good, A. C.; Kuntz, I. D. J Mol Recognit 1996, 9 , 175.

4. Read, R. J.; Hart, T. N.; Cummings, M. D. Ness, S. R. Supramol Chem 1995, 6, 135.

5. Leach, A. R. J Mol Biol 1994, 235, 345.

6. Blaney, J. M.; Dixon, J. S. Perspect Drug Dis Design 1993, 1, 301.

7. Katchalski-Katzir, E.; Shariv, I.; Eisenstein, M.; Friesem, A. A.; Aflalo, C.; Vakser, I. A. Proc Natl Acad Sci USA 1992, 89, 2195.

8. Gabb, H. A.; Jackson, R. M.; Sternberg, M. J. E. J Mol Biol 1997, 272, 106.

9. Kuntz, I. D.; Blaney, J. M.; Oatley, S. J.; Langridge, R.; Ferrin, T. A. J Mol Biol 1982, 161, 269.

10. Jiang, F.; Kim, S. H. J Mol Biol 1991, 219, 79.

11. Desmet, J.; Wilson, I. A.; Joniau, M.; DeMaeyer, M.; Lasters, I. FASEB J 1997, 11, 164.

12. Morris, G. M.; Goodsell, D. S.; Huey, R.; Olson, A. J. J Comput Aid-Mol Design 1996, 10, 293.

13. Peters, K. P.; Fauck, J.; Frommel, C. J Mol Biol 1996, 256, 201.

14. Laskowski, R. A.; Luscombe, N. M.; Swindells, M. B.; Thornton, J. M. Protein Sci 1996, 5, 2438.

15. Weng, Z. P.; Vajda, S.; Delisi, C. Protein Sci 1996, 5, 614.

16. Robert, C. H.; Janin, J. J Mol Biol 1998, 283, 1037.

17. Muegge, I.; Martin, Y. C. J Med Chem 1999, 42, 791.

18. Norel, R.; Lin, S. L.; Wolfson, H. J.; Nussinov, R. J Mol Biol 1995, 252, 263.

19. Norel, R.; Petrey, D.; Wolfson, H. J.; Nussinov, R. Protein Struct Funct Genet 1999, 36, 307.

20. Palma, P. N.; Krippahl, L.; Wampler, J. E.; Moura, J. J. G. Protein Struct Funct Genet 2000, 39, 372.

21. Vakser, I. A. Protein Eng 1996, 9, 37.

22. Vakser, I. A. Biopolymers 1996, 39, 455.

23. Vakser, I. A.; Matar, O. G.; Lam, C. F. Proc Natl Acad Sci USA 1999, 96, 8477.

24. Vakser, I. A. Protein Struct Funct Genet 1997, 1, 226.

25. Vakser, I. A. Protein Eng 1995, 8, 371.

26. Ritchie, D. W.; Kemp, G. J. L. Protein Struct Funct Genet 2000, 39, 178.

27. Trosset, J. Y.; Scheraga, H. A. Proc Natl Acad Sci USA 1998, 95, 8011.

28. Skolnick, J.; Kihara, D. Proteins 2001, 42, 319.

29. Kolinski, A.; Rotkiewicz, P.; Ilkowski, B.; Skolnick, J. Proteins 1999, 37, 592.

30. Bernstein, F. C.; Koetzle, T. F.; Williams, G. J. B.; Meyer, E. F., Jr.; Brice, M. D.; Rodgers, J. R.; et al. J Mol Biol 1977, 112, 535.

31. Sharff, A. J.; Rodseth, L. E.; Spurlino, J. C.; Quiocho, F. A. Biochemistry $1992,31,10657$.

32. Schulz, G. E. Faraday Discuss 1992, 93, 85.

33. Fischer, D.; Elofsson, A.; Rice, D.; Eisenberg, D. Pac Symp Biocomput 1996, 300 .

34. http://www.doe-mbi.ucla.edu/people/fischer/BENCH/table1.html. UCLA. 1996, Abstract.

35. Benning, M. M.; Smee, A. F.; Wells, M. A.; Holden, H. M. J Mol Biol 1992, 228, 208.

36. Meyer, M.; Wilson, P.; Schomburg, D. J Mol Biol 1996, 264, 199. 